\title{
Detection of atomic oxygen in a plasma-assisted flame via backward lasing technique
}

\author{
PENGJI DING ${ }^{1, *}$, MARIA RUCHKINA ${ }^{1}$, DAVIDE DEL CONT-BERNARD ${ }^{2}$, ANDREAS EHN $^{1}$, \\ DEANNA A. LACOSTE ${ }^{2}$, AND JOAKIM BOOD ${ }^{1}$
}

\author{
${ }^{1}$ Division of Combustion Physics, Department of Physics, Lund University, Box 118, SE-221 00 Lund, Sweden \\ ${ }^{2}$ Clean Combustion Research Center, King Abdullah University of Science and Technology, Thuwal, 23955-6900, Saudi Arabia \\ *Corresponding author: pengji.ding@forbrf.lth.se
}

Compiled October 18, 2019

In this work, we have investigated 845-nm lasing generation in atomic oxygen, present in a lean methane-air flame, using 2-photon pumping with femtosecond 226$\mathrm{nm}$ laser pulses, particularly focusing on the impact of nanosecond repetitively pulsed glow discharges forcing on the backward lasing signal. Characterizations of the backward lasing pulse, in terms of its spectrum, beam profile, pump pulse energy dependence and divergence, were conducted to establish the presence of lasing. With plasma forcing of the flame, the backward lasing signal was observed to be enhanced significantly, $\sim 50 \%$. The vertical concentration profile of atomic oxygen was revealed by measuring the backward lasing signal strength as a function of heights in the flame. The results are qualitatively consistent with results obtained with 2-dimensional femtosecond two-photon-absorption laser-induced fluorescence (fsTALIF), suggesting that the backward lasing technique can be a useful tool for studies of plasma-assisted combustion processes, particularly in geometries requiring single-ended standoff detection. () 2019 Optical Society of America

\section{http://dx.doi.org/10.1364/ao.XX.XXXXXX}

By focusing a pump laser onto a tiny volume of atoms or molecules, highly directional emission or "lasing" along the pump laser beam, in both the forward and backward directions, may emerge at a wavelength different from that of the pump laser. The backward lasing travels back towards the pump laser and thus samples the intervening medium as it returns. As such, backward lasing could potentially provide single-ended remote sensing of atomic/molecular species that are difficult or impossible to study with conventional laser-based techniques. It could be used to monitor atmospheric pollutants, trace gases remaining indicative of hazardous substances, such as explosive devices, or investigate combustion conditions in furnaces and engines, just to give a few examples. Driven by its very promising potential to manage the aforementioned applications, backward-lasing techniques has lately been a topic of intense research within the research communities of ultrafast optics and laser-based diagnostics. The pioneering works on backward lasing in air by Luo et al [1] and Dogariu et al [2] have been followed by a significant number of papers demonstrating various measurement concepts and applications; see e.g. [3-10].

These vivid activities were inspired by earlier works in flames, in which backward lasing of important intermediate species, such as $\mathrm{O}, \mathrm{H}$, and $\mathrm{C}$ atoms, have been observed with nanosecond (ns) laser pumping [11-13]. However, the observed backward lasing pulses had a ns-scale duration with spiky temporal profiles, resulting in poor and ambiguous spatial resolution, severely limiting its diagnostic potential. In addition, the high UV-pulse energy required for efficient pumping, typically a few millijoules, makes the concept prone to photolytic interferences. To overcome these difficulties, we recently demonstrated backward lasing from atomic hydrogen in flames based on femtosecond (fs) laser pumping, which produced backward lasing pulses of picosecond-scale duration and with smooth temporal profiles $[14,15]$. These characteristics of the lasing pulses, together with highly time-resolved detection, allow single-ended measurements with spatial resolution on the millimeter scale for the backward lasing technique. The potential of the method for combustion diagnostics has recently been demonstrated in laboratory flames, where it was shown that the hydrogen signal from two reaction zones separated by $2 \mathrm{~mm}$ can be resolved and that the lasing signal is strong enough for single-shot measurements $[14,16]$.

In this work, we apply the backward lasing technique (BLT) to assess the impact of plasma induced by Nanosecond Repetitively Pulsed (NRP) glow discharge on a flame in terms of the distribution of atomic oxygen, and compare the results with the corresponding results obtained with fs-TALIF. The oxygen atom was chosen to be studied since it plays an important role in the kinetics of hydrocarbon oxidation, which also makes it a key species and hence the interest of plasma-assisted combustion applications [17]. For detection of $\mathrm{O}$ atoms, two-photon resonant excitation from the $2 p^{3} P$ ground state to the $3 p^{3} P$ excited state is carried out using 226-nm fs laser pulses. De-excitation then takes place from the $3 p^{3} P$ to the $3 s^{3} S$ state, corresponding to a photon wavelength of $845 \mathrm{~nm}$ (see Fig. 1(a)). In addition to relaxation through spontaneous emission, i.e. fluorescence, the highly intense pumping achieved with fs laser pulses can create population inversion between the two states, generating 

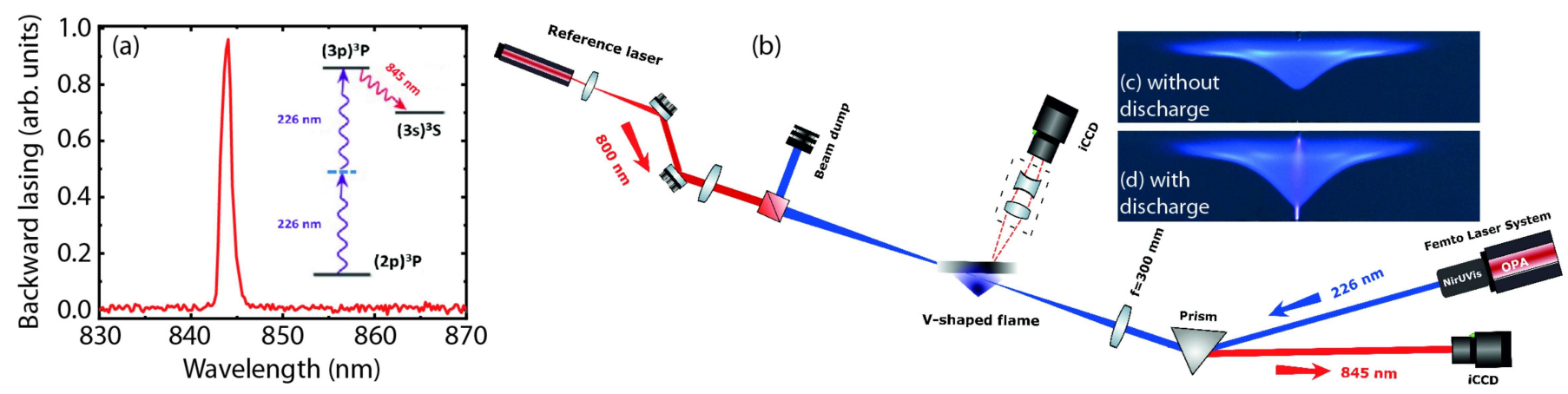

Fig. 1. (a) Spectrum of $845 \mathrm{~nm}$ lasing emission in the backward direction measured with a low-resolution spectrometer. The insert shows the two-photon resonant excitation scheme of the $\mathrm{O}$ atom and subsequent emission at $845 \mathrm{~nm}$. (b) Schematic illustration of the experimental setup. Inserts (a) and (b) show the side view of the flame without and with plasma forcing produced with $10 \mathrm{kHz}$, $8.0 \mathrm{kV}$ glow discharge, respectively. The photos were taken with an ordinary digital camera.

stimulated emission, here referred to as lasing, which was originally observed in sub-atmospheric flames by Aldén et al. [11]. Characterization of the backward lasing pulses from $\mathrm{O}$ atoms, including emission spectrum, spatial profile, and divergence, was first performed to confirm the occurrence of lasing. It was found that the backward lasing signal increased significantly ( 54\%) with $10 \mathrm{kHz}, 8.5 \mathrm{kV}$ glow discharge forcing on the flame. A comparison with 2D fs-TALIF imaging shows that both techniques can provide reliable vertical profiles of $\mathrm{O}$ atoms along the discharge path.

The experimental setup is shown in Fig. 1(b). A wavelengthtunable femtosecond laser system was used to provide $125 \mathrm{fs}$ laser pulses at $226 \mathrm{~nm}$ wavelength with a maximum pulse energy of $\sim 200 \mu \mathrm{J}$. The beam size is about $5 \mathrm{~mm}$ in diameter, and the spectral bandwidth is about $2 \mathrm{~nm}$ (full width at half maximum, FWHM). The $226 \mathrm{~nm}$ laser beam is firstly sent through a dispersive prism and then focused with an $f=300 \mathrm{~mm}$ spherical $\mathrm{CaF}_{2}$ lens into a V-shaped flame, where atomic oxygen is naturally present. The flame is a lean methane-air flame, confined by a co-flow of nitrogen. It stabilizes between the nozzle and a stagnation quartz plate in a plasma-assisted combustion (PAC) burner (see Fig. 3.8 in [18]). The equivalence ratio is fixed at 0.76 (i.e. a fuel-lean flame). Two pin electrodes ( $9 \mathrm{~mm}$ gap) are placed in such a way that the pin-to-pin glow discharge passes through the symmetry axis of the flame. The insert photos in Fig. 1(b1) and (b2) show direct imaging of the flame without and with NRP glow discharge forcing, respectively. The discharges are produced by high-voltage pulses of $10 \mathrm{~ns}$ duration, $8.0 \mathrm{kV}$ amplitude between the pin anode in the burner and the grounded pin electrode of the quartz plate, applied at a repetition rate of $10 \mathrm{kHz}$.

The focusing of $226 \mathrm{~nm}$ laser pulses into the flame creates a narrow filamentary excitation volume of $\mathrm{O}$ atoms, from where $845 \mathrm{~nm}$ lasing occurs in both the forward and backward direction. The backward lasing beam is collimated by the focusing lens, and then separated from the incident $226 \mathrm{~nm}$ laser beam by a prism. Finally, the backward lasing signal is detected by an intensified CCD camera (iCCD, Princeton Instruments, PIMAX4 1024f) or a fiber spectrometer (not shown in Fig. 1(b)). A reference laser beam at $800 \mathrm{~nm}$ was used for alignment of the setup, particularly to roughly predict the optical path of the $845 \mathrm{~nm}$ backward lasing beam. For 2D fs-TALIF measurements, a $\sim 5$-mm-wide laser sheet was created with a cylindrical lens $(f=500 \mathrm{~mm})$ and focused into the flame. The iCCD camera, equipped with a $135 \mathrm{~mm} \mathrm{f/2.8} \mathrm{lens} \mathrm{(Nikon)} \mathrm{and} \mathrm{two} \mathrm{bandpass}$ interference filters, were used to record the fluorescence images of atomic oxygen in the side direction. The camera gate width was $4 \mathrm{~ns}$, and each fs-TALIF image is an accumulation of 250 shots.

We started experiments in the unperturbed flame, i.e. without plasma forcing. To establish the presence of lasing, the signal emitted backwards along the excitation volume is compared to the fluorescence signal detected from the side direction. Figure 2(a) shows both the backward emission and the fluorescence signal as a function of the $226 \mathrm{~nm}$ pump laser pulse energy. If the backward emission is incoherent fluorescence, then these two measurements should give similar scaling behavior. On the other hand, if the backward emission indeed is lasing due to stimulated emission, then the shapes of the curves will be different since lasing requires pump pulse energies above a certain threshold to occur. As can be seen in Fig. 2(a), the fluorescence signal intensity increases gradually with pump pulse energy. Power function fitting to the data suggests a power index of $1.8 \pm 0.3$, which is in consistent with two-photon process. On the other hand, the backward emission intensity is following a much faster pulse energy dependence, showing clearly different low-pump-intensity behavior from that of the fluorescence signal, similar to what has been reported in $[2,11]$. The dependence curve also shows the signature of a threshold at $\sim 45 \mu \mathrm{J}$.

At a position of $\sim 1.5 \mathrm{~m}$ away from the PAC burner, the iCCD camera is used to capture the profile of the backward $845 \mathrm{~nm}$ lasing beam. The divergence of the backward lasing beam is determined by measuring its size at two different positions along the propagation path. It is found that the backward lasing is very localized with a small beam divergence, another indicative signature of lasing effect. A typical result, displayed in Fig. 2(b), shows a Gaussian-shaped spatial profile with a divergence of $\sim 5$ mrad. However, it should be noticed that the spatial mode is dynamic, mostly due to pulse-to-pulse energy variations of the $226 \mathrm{~nm}$ pump laser. With a relatively stable pump pulse energy of $108.0 \pm 5.3 \mu \mathrm{J}$, the divergence of the backward lasing beam is recorded continuously over 100 laser pulses, and the results are shown in Fig. 2(c). The mean divergence is found to be $4.7 \mathrm{mrad}$ with a standard deviation of $0.7 \mathrm{mrad}$.

Figure 3 shows the beam divergence of the backward lasing pulse measured at different vertical positions $y$ in the flame. The $y=0$ is defined as the position of the upper electrode, and the positive $y$ points to the lower electrode. For $y<2.0 \mathrm{~mm}$, 

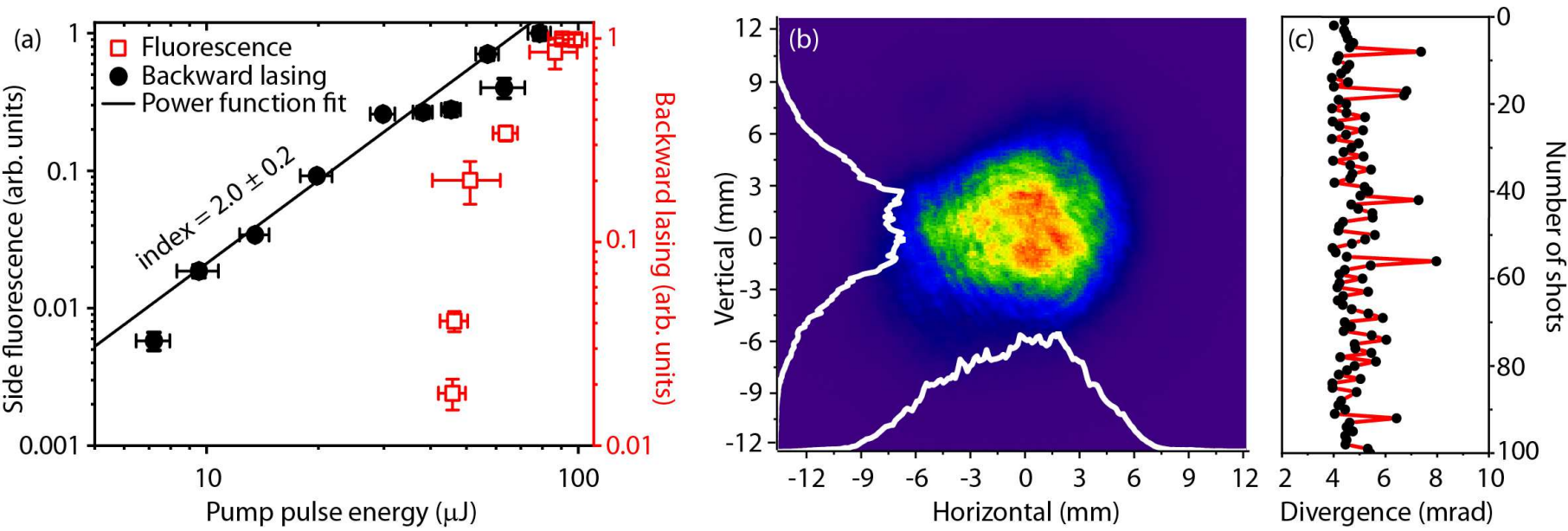

Fig. 2. (a) The pump pulse energy dependence of the fluorescence signal, and backward lasing signals at $845 \mathrm{~nm}$. (b) Far-field, single-shot backward $845 \mathrm{~nm}$ lasing beam measured $1.5 \mathrm{~m}$ away from the $226 \mathrm{~nm}$ laser focusing point in the flame. The white solid lines depict the horizontal and vertical beam profiles. (c) The variation of the lasing beam divergence over 100 laser shots.
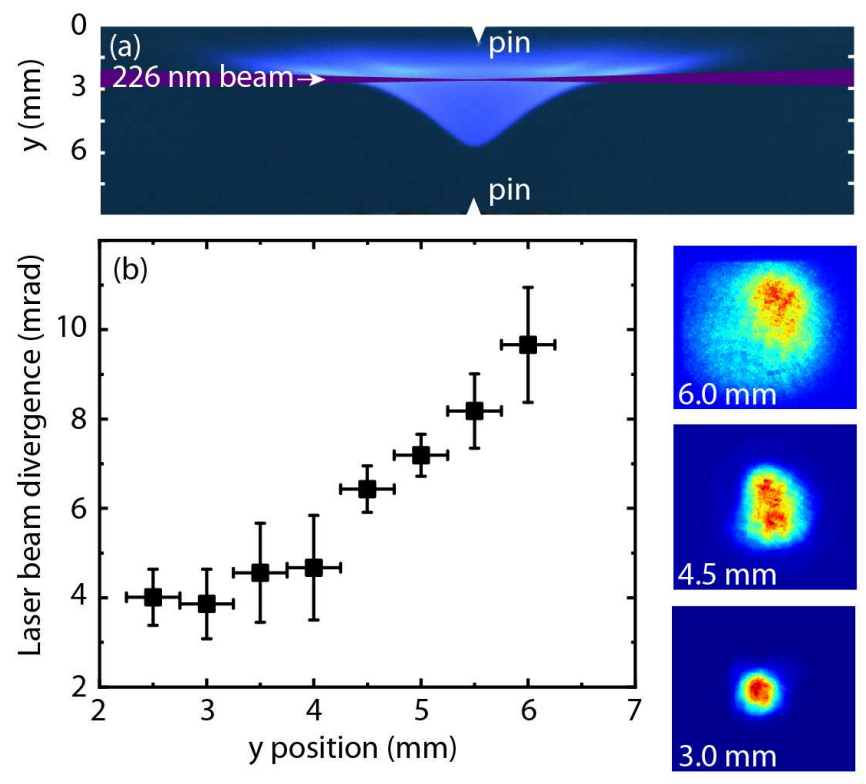

Fig. 3. (a) Schematic illustration of focusing 226-nm laser into the flame at different $y$ positions. (b) The beam divergence of the backward $845 \mathrm{~nm}$ lasing as a function of $y$. The lasing beam images with $12 \mathrm{mrad} \times 12$ mard size taken by the iCCD camera for $y=3.0,4.5$ and $6.0 \mathrm{~mm}$ are also shown in the inserts, respectively.

the $226 \mathrm{~nm}$ pump laser is blocked by the quartz plate. Below the flame tip where $y=6 \mathrm{~mm}$, there is no detectable lasing signal since there are no $\mathrm{O}$ atoms in the cold gases. One can see that the divergence of the backward lasing beam gradually increases with $y$. Backward lasing beam images recorded at 3 different vertical positions (3, 4.5 and $6.0 \mathrm{~mm})$ are shown on the right-hand-side of Fig. 3, from which it can be seen that the enlargement of the lasing beam size with increasing $y$ is significant. This is due to the fact that the length of gain medium created by 226-nm laser becomes shorter for larger $y$, i.e. closer to the flame tip, and the lasing beam divergence inversely depends on the gain medium length [19], which turns out that the lasing beam divergence increases with $y$.

It is notable in Fig. 1 that the flame is pushed down to the bottom electrode under NRP discharge-induced plasma forcing. Two-dimensional fs-TALIF imaging of atomic oxygen is conducted to measure its spatial distribution in the flame without and with plasma forcing. The results are shown in Fig. 4 (a) and (b), respectively. The image shown in Fig. 4(b) is recorded $\sim 1 \mu \mathrm{s}$ after the discharge event. In addition to the push-down of the $\mathrm{O}$-atom distribution, the fluorescence signal is enhanced significantly by the discharge forcing at the flame tip and in the vicinity of the flame front. Hence, the plasma forcing implies enhanced production of $\mathrm{O}$ atoms in the reaction zone of the flame. In addition, a faint fluorescence signal is noticeable in the discharge channel connecting the flame tip and the anode, which could come from atomic oxygen produced by plasma and post-discharge chemical reactions. In order to compare the fluorescence signals with the corresponding backward lasing signals, the fluorescence signal in the fs-TALIF image is integrated along the propagation direction of the pump laser and then plotted as a function of the vertical positions, as shown in Fig. 4(c) and (d).

To investigate the impact of plasma forcing on the flame using the backward lasing technique, the $226 \mathrm{~nm}$ pump laser has been focused at different heights in the flame, whereupon the backward lasing signal yield has been measured without and with plasma forcing. The results are shown in Fig. 4(e) and (f). The maximal backward lasing signal increased by $\sim 54 \%$ during plasma forcing compared to the corresponding signal intensity in the unperturbed flame. This result agrees with the vertical profiles of the integrated fluorescence shown in Fig. 4(c) and (d) A slight vertical peak shift of the lasing signal can be noticed as compared with the integrated fluorescence peak, which is due to the increased length of gain medium with shorter vertical distances. The maximal fluorescence signal exhibits a 58\% increase with plasma forcing. Similar percentages of increase between the fluorescence and backward lasing signals strongly indicate that these two methods have similar concentration dependence. This is because the backward lasing effect is close to the saturation regime of its optical gain with a maximum 226-nm pulse energy of $\sim 100 \mu \mathrm{J}$. An even closer agreement is expected 

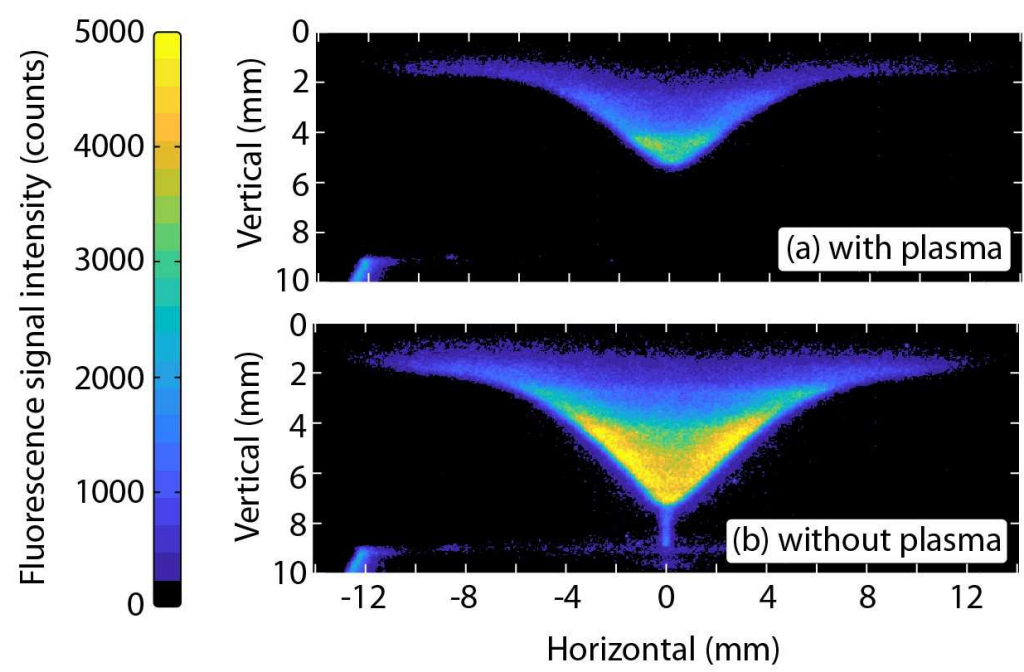

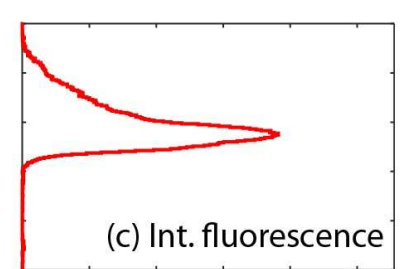

(c) Int. fluorescence

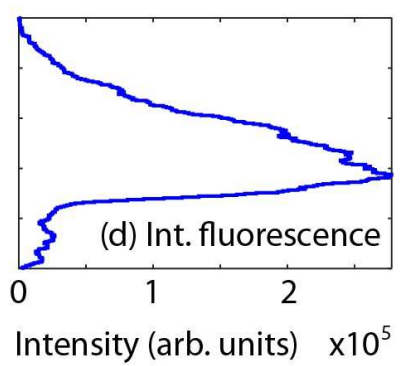

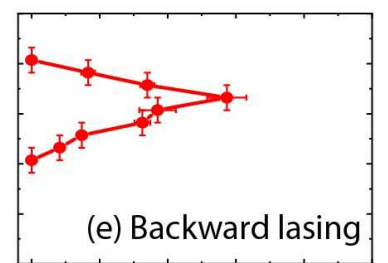

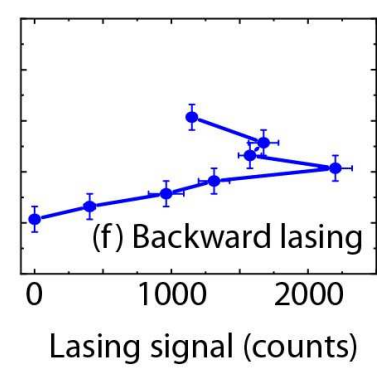

Fig. 4. $2 \mathrm{D}$ fs-TALIF images of atomic oxygen in (a) the base flame and (b) the flame under the glow discharge forcing (10 kHz repetition rate, $8.0 \mathrm{kV}$ voltage). (c) and (d) The integrated fluorescence profiles of these two fluorescence images over the vertical direction, respectively. (e) and (f) Backward $845 \mathrm{~nm}$ lasing signal of atomic oxygen measured at different heights in the base flame and in the flame under the glow discharge forcing respectively. The 226-nm laser pulse energy used in these measurements is $\sim 100 \mu \mathrm{J}$.

with even higher 226-nm pulse energies.

To summarize, we generated bidirectional $845 \mathrm{~nm}$ lasing emission of atomic oxygen by focusing femtosecond $226 \mathrm{~nm}$ laser pulses into a V-shaped lean methane-air flame. Particular focus was put on the backward lasing signal due to its potential applications for standoff remote sensing. To establish the presence of the lasing, lasing beam profile, divergence, and pump pulse energy dependence of the backward lasing were measured. The impact of NRP glow discharge forcing on the backward lasing strength was also investigated, and it was found to significantly enhance the signal strength by $\sim 54 \%$. By comparing the results from backward lasing with 2D fs-TALIF images of atomic oxygen, it was found that the vertical concentration profile can be reliably measured by the backward lasing technique. In the nearsaturation regime, backward lasing shows similar concentration dependence as laser-induced fluorescence. These results suggest that the backward lasing technique could be a useful diagnostic tool for plasma-assisted combustion studies, particularly in measurement geometries requiring single-ended standoff measurements.

\section{FUNDING.}

This research work was sponsored by the King Abdullah University of Science and Technology (KAUST), the Knut and Alice Wallenberg Foundation, the European Research Council (ERC), The Swedish Foundation for Strategic Research (SSF), the Swedish Research Council (VR) and the Swedish Energy Agency through the Centre for Combustion Science and Technology (CECOST).

\section{DISCLOSURES.}

The authors declare no conflicts of interest.

\section{REFERENCES}

1. Q. Luo, W. Liu, and S. Chin, Appl. Phys. B 76, 337 (2003).

2. A. Dogariu, J. B. Michael, M. O. Scully, and R. B. Miles, Sci. (New York, N.Y.) 331, 442 (2011).
3. P. R. Hemmer, R. B. Miles, P. Polynkin, T. Siebert, A. V. Sokolov, P. Sprangle, and M. O. Scully, Proc. Natl. Acad. Sci. 108, 3130 (2011).

4. A. J. Traverso, R. Sanchez-Gonzalez, L. Yuan, K. Wang, D. V. Voronine, A. M. Zheltikov, Y. Rostovtsev, V. A. Sautenkov, A. V. Sokolov, S. W. North, and M. O. Scully, Proc. Natl. Acad. Sci. 109, 15185 (2012).

5. A. Laurain, M. Scheller, and P. Polynkin, Phys. Rev. Lett. 113, 253901 (2014).

6. S. Mitryukovskiy, Y. Liu, P. Ding, A. Houard, and A. Mysyrowicz, Opt. Express 22, 12750 (2014).

7. P. Ding, S. Mitryukovskiy, A. Houard, E. Oliva, A. Couairon, A. Mysyrowicz, and Y. Liu, Opt. Express 22, 29964 (2014).

8. P. N. Malevich, R. Maurer, D. Kartashov, S. Ališauskas, A. A. Lanin, A. M. Zheltikov, M. Marangoni, G. Cerullo, A. Baltuška, and A. Pugžlys, Opt. Lett. 40, 2469 (2015).

9. A. Dogariu and R. B. Miles, Opt. Express 24, A544 (2016).

10. P. Polynkin and Y. Cheng, Air Lasing, 208 (Springer International Publishing, 2018).

11. M. Aldén, U. Westblom, and J. E. M. Goldsmith, Opt. Lett. 14, 305 (1989).

12. J. E. M. Goldsmith, J. Opt. Soc. Am. B 6, 1979 (1989).

13. U. Westblom, S. Agrup, M. Aldén, H. M. Hertz, and J. E. M. Goldsmith, Appl. Phys. B 50, 487 (1990).

14. P. Ding, M. Ruchkina, Y. Liu, M. Alden, and J. Bood, Opt. Lett. 43, 1183 (2018).

15. P. Ding, M. Ruchkina, Y. Liu, M. Alden, and J. Bood, Opt. Lett. 44, 2374 (2019).

16. M. Ruchkina, P. Ding, A. Ehn, M. Aldén, and J. Bood, Proc. Combust. Inst. 37, 1281 (2019).

17. C. K. Law, Combustion Physics (Cambridge University Press, 2006).

18. S. Heitz, "Interaction mechanisms between nanosecond repetitively pulsed plasma discharges and laminar reactive flows," Theses, Université Paris-Saclay ; Technische Universität (Berlin) (2017).

19. G. I. Peters and L. Allen, J. Phys. A: Gen. Phys. 5, 546 (1972). 


\section{FULL REFERENCES}

1. Q. Luo, W. Liu, and S. Chin, "Lasing action in air induced by ultra-fast laser filamentation," Appl. Phys. B 76, 337-340 (2003).

2. A. Dogariu, J. B. Michael, M. O. Scully, and R. B. Miles, "High-gain backward lasing in air." Sci. (New York, N.Y.) 331, 442-5 (2011).

3. P. R. Hemmer, R. B. Miles, P. Polynkin, T. Siebert, A. V. Sokolov, P. Sprangle, and M. O. Scully, "Standoff spectroscopy via remote generation of a backward-propagating laser beam," Proc. Natl. Acad. Sci. 108, 3130-3134 (2011).

4. A. J. Traverso, R. Sanchez-Gonzalez, L. Yuan, K. Wang, D. V. Voronine, A. M. Zheltikov, Y. Rostovtsev, V. A. Sautenkov, A. V. Sokolov, S. W. North, and M. O. Scully, "Coherence brightened laser source for atmospheric remote sensing," Proc. Natl. Acad. Sci. 109, 15185-15190 (2012).

5. A. Laurain, M. Scheller, and P. Polynkin, "Low-Threshold Bidirectional Air Lasing," Phys. Rev. Lett. 113, 253901 (2014).

6. S. Mitryukovskiy, Y. Liu, P. Ding, A. Houard, and A. Mysyrowicz, "Backward stimulated radiation from filaments in nitrogen gas and air pumped by circularly polarized $800 \mathrm{~nm}$ femtosecond laser pulses," Opt. Express 22, 12750 (2014).

7. P. Ding, S. Mitryukovskiy, A. Houard, E. Oliva, A. Couairon, A. Mysyrowicz, and Y. Liu, "Backward lasing of air plasma pumped by circularly polarized femtosecond pulses for the sake of remote sensing (black)," Opt. Express 22, 29964-29977 (2014).

8. P. N. Malevich, R. Maurer, D. Kartashov, S. Ališauskas, A. A. Lanin, A. M. Zheltikov, M. Marangoni, G. Cerullo, A. Baltuška, and A. Pugžlys, "Stimulated raman gas sensing by backward uv lasing from a femtosecond filament," Opt. Lett. 40, 2469-2472 (2015).

9. A. Dogariu and R. B. Miles, "Three-photon femtosecond pumped backwards lasing in argon," Opt. Express 24, A544-A552 (2016).

10. P. Polynkin and Y. Cheng, Air Lasing, 208 (Springer International Publishing, 2018).

11. M. Aldén, U. Westblom, and J. E. M. Goldsmith, "Two-photon-excited stimulated emission from atomic oxygen in flames and cold gases," Opt. Lett. 14, 305-307 (1989).

12. J. E. M. Goldsmith, "Two-photon-excited stimulated emission from atomic hydrogen in flames," J. Opt. Soc. Am. B 6, 1979-1985 (1989).

13. U. Westblom, S. Agrup, M. Aldén, H. M. Hertz, and J. E. M. Goldsmith, "Properties of laser-induced stimulated emission for diagnostic purposes," Appl. Phys. B 50, 487-497 (1990).

14. P. Ding, M. Ruchkina, Y. Liu, M. Alden, and J. Bood, "Femtosecond two-photon-excited backward lasing of atomic hydrogen in a flame," Opt. Lett. 43, 1183-1186 (2018).

15. P. Ding, M. Ruchkina, Y. Liu, M. Alden, and J. Bood, "Gain mechanism of femtosecond two-photon-excited lasing effect in atomic hydrogen," Opt. Lett. 44, 2374-2377 (2019).

16. M. Ruchkina, P. Ding, A. Ehn, M. Aldén, and J. Bood, "Single-shot, spatially-resolved stand-off detection of atomic hydrogen via backward lasing in flames," Proc. Combust. Inst. 37, 1281 - 1288 (2019).

17. C. K. Law, Combustion Physics (Cambridge University Press, 2006).

18. S. Heitz, "Interaction mechanisms between nanosecond repetitively pulsed plasma discharges and laminar reactive flows," Theses, Université Paris-Saclay ; Technische Universität (Berlin) (2017).

19. G. I. Peters and L. Allen, "Amplified spontaneous emission. IV. beam divergence and spatial coherence," J. Phys. A: Gen. Phys. 5, 546-554 (1972). 